\section{Medullary carcinoma of the thyroid presenting as multifocal bronchial carcinoid tumour}

\author{
J E Clague, M G Pearson, A Sharma, \\ W Taylor
}

\begin{abstract}
A 21 year old man presented with diarrhoea and flushing after meals and later developed miliary shadowing on his chest radiograph. Multifocal bronchial carcinoid tumour was diagnosed initially, but at necropsy metastatic medullary carcinoma of the thyroid was found. Multifocal bronchial carcinoid tumour should not be accepted as a primary diagnosis without first excluding medullary carcinoma of the thyroid because of the need to screen relatives of affected patients.
\end{abstract}

Multifocal bronchial carcinoid tumour or carcinoid lung has been proposed as a primary pulmonary diagnosis. ${ }^{1}$ We present a case, similar to the one described in the initial report, in which the final diagnosis was metastatic medullary carcinoma of the thyroid, a histologically indistinguishable condition.

\section{Case report}

A 21 year old man presented with flushing after meals and diarrhoea. Extensive investigations, including thyroid function tests (thyroxine, thyroid stimulating hormone) and hormone assays for gastrin, glucagon, vasoactive intestinal peptide, neurotensin, and pancreatic polypeptide, gave normal results, as did urine analysis for 5-hydroxyindoleacetic acid and vanillylmandelic acid. A small bowel meal and follow through showed only intestinal hurry; sigmoidoscopy and rectal biopsy showed nothing abnormal.

The patient was lost to follow up for two years, after which he returned complaining of pain in the right iliac fossa and weight loss (10 kg in one year). A marfanoid habitus and wasting and weakness of proximal muscles were noted. He had no chest symptoms. Abdominal examination showed guarding in the right iliac fossa. A chest radiograph showed miliary opacities (fig 1). Pulmonary function testing gave results within normal limits apart from reduced transfer factor for carbon monoxide ( $60 \%$ predicted). Transbronchial biopsy showed nodular foci of amyloid in the bronchial, vascular, and alveolar walls, confirmed by Congo red staining and electron microscopy. Between the amyloid nodules were collections of regular cells with rounded or oval nuclei and

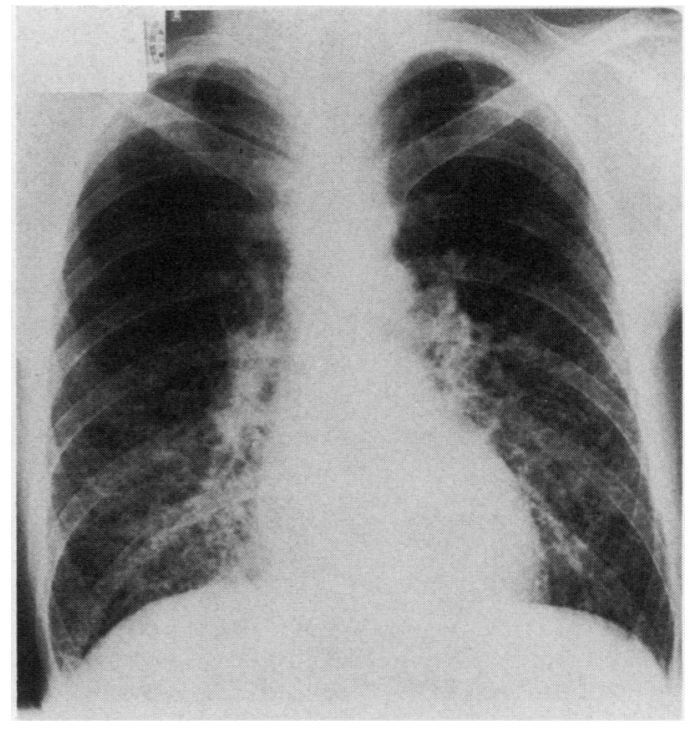

Figure 1 Chest radiograph showing miliary opacities.

moderate amounts of eosinophilic cytoplasm (fig 2). Immunohistochemical staining with neurone specific enolase indicated that the cells were producing polypeptide and a diagnosis of multifocal bronchial carcinoid tumour of the lung was considered.

The patient subsequently developed a mass in the right iliac fossa, rectal bleeding, and a raised white cell count. At laparotomy caecal diverticulitis and abscesses were found. $\mathrm{He}$ underwent a hemicolectomy and died seven weeks later of persistent abdominal sepsis.

At necropsy a medullary carcinoma of the thyroid was found. The right lobe and isthmus of the thyroid were replaced by a hard waxy mass of tumour invading adjacent thyroid tissue and extrathyroid fat. Cervical, mediastinal, and abdominal lymph node metastases were present. The lungs contained multiple miliary tumour nodules, mainly less than $1 \mathrm{~mm}$ in diameter but up to $2 \mathrm{~mm}$ on the pleural surface. The metastases in the mediastinal lymph nodes contained large, waxy amyloid masses. Amyloid was found only in relation to the primary tumour and metastases. Tumour specimens gave positive immunohistochemical reactions only for calcitonin, calcitonin gene related peptide, chromagranin, and protein gene product. Examination of the parathyroid and adrenal glands showed no evidence of tumour.

\section{Discussion}

In 1976 Skinner and Ewen reported "multifocal bronchial carcinoid tumour" in a young man with flushing, diarrhoea, and weight loss, ${ }^{1}$ who died about 10 years later without a postmortem examination (Ewen, personal communication). Our case is strikingly similar. One other report of miliary pulmonary carcinoid tumour with amyloid has been published, ${ }^{2}$ the authors of which suggest the possibility of tumour origin from the $\mathrm{C}$ cells of the thyroid.
Fazakerley Hospital

J E Clague

M G Pearson

W Thaylor

Reprint requests to:

Dr Clague

Accepted 18 April 1990 


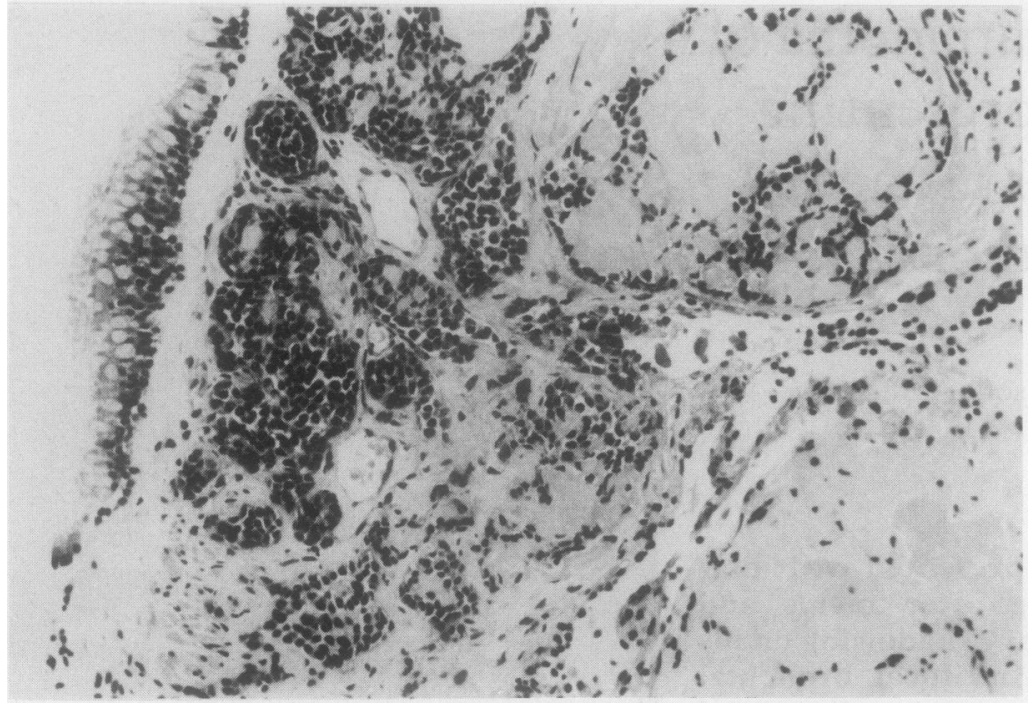

Figure 2 Bronchial wall with ciliated epithelium on the left and pale staining amyloid on the right. ${ }^{\star} N$ Nests and cords of dark-staining tumour cells are present beneath the surface epithelium and between the nodules of amyloid. (Haematoxylin and eosin.)

The histological appearance of the lesion previously described as miliary pulmonary carcinoid tumour with amyloid is identical to that of metastatic medullary carcinoma of the thyroid. This tumour may present with symptoms of the carcinoid syndrome and pulmonary metastases are recognised in disseminated disease. ${ }^{3}$ Medullary carcinoma of the thyroid contains calcitonin but so may bronchial carcinoid, and both lesions may contain amyloid. ${ }^{4}$

Medullary carcinoma accounts for about $10 \%$ of thyroid tumours and the course of disease is variable. ${ }^{3}$ About a quarter of cases are familial. $^{35}$ Inherited as an autosomal dominant condition, it may form part of the multiple endocrine neoplasia syndrome type 2. A marfanoid habitus, myopathy, and colonic diverticulosis have been associated with the type $2 b$ variant of this syndrome. ${ }^{6}$ Even in cases that are apparently sporadic (probably because gene expression is variable) $10-15 \%$ of cases have the familial multiple endocrine neoplasia syndrome. ${ }^{5}$ Bilateral tumour and $\mathrm{C}$ cell hyperplasia in the thyroid gland may indicate familial disease and screening of relatives by means of stimulated calcitonin measurement may allow early detection and curative surgery. ${ }^{356}$ The relatives of our patient are currently undergoing screening. We believe that the patient reported by Skinner and Ewen ${ }^{1}$ may have had medullary carcinoma of the thyroid. Multifocal bronchial carcinoid should not be accepted as a primary lung lesion without first excluding metastatic medullary carcinoma of the thyroid.

1 Skinner C, Ewen SWB. Carcinoid lung: pulmonary infiltration by a multifocal bronchial carcinoid. Thorax 1976; 31:212-9.

2 Cain H, Egner E. Metastasizing amyloid producing carcinoma of the APUD series. Verh Dtsch Pathol 1977;61: 314-8.

3 Saad MF, Ordonez NG, Rashid RK, et al. Medullary carcinoma of the thyroid. A study of clinical features and prognostic factors in 161 patients. Medicine (Baltimore) 1984;63:319-42.

4 Al-Kaisi N, Abdul-Karim FW, Mendelsohn G, et al. Bronchial carcinoid tumour with amyloid stroma. Arch Pathol Lab Med 1988;112:211-4.

5 Ponder BAJ, Ponder MA, Coffey R, et al. Risk estimation and screening in families of patients with medullary thyroid carcinoma. Lancet 1988;i:397-400.

6 Carney JA, Sizemore GW, Hayles AB. Multiple endocrine neoplasia, type 2 b. In: Ioachim HL, ed. Pathology annual 1978. Vol 8. New York: Raven Press, 1978:105-53.
Anti-basement membrane antibody disease with severe pulmonary haemorrhage and
normal renal function

\footnotetext{
Department of

Medicine

A Tobler

E Schürch

Institute of Pathology H J Altermatt

Division of

Respiratory Diseases

V Im Hoff

Inselspital, University

of Berne, Berne,

Switzerland

Address for reprint requests: Dr A Tobler, Department of Medicine, Inselspital,

CH 3010 Berne, Switzerland.

Accepted 20 June 1990
}

\author{
Abstract \\ A case of anti-basement membrane dis- \\ ease with severe pulmonary haemo- \\ rrhage and normal renal function is \\ reported.
}

In 1919 Goodpasture described a syndrome of glomerulonephritis and pulmonary haemorrhage. ${ }^{1}$ Half a century later both manifestations were shown to be induced by circulating autoantibodies that reacted with alveolar and glomerular basement membranes. ${ }^{2}$ We present a case with severe pulmonary haemorrhage mediated by anti-basement membrane antibody in which renal function was normal.

\section{Case report}

A previously healthy 20 year old cigarette smoking farmer was referred complaining of dyspnoea and progressive haemoptysis of four days' duration after accidental exposure to fumes of fungicides containing metalaxyl $(8 \%)$ and mancozeb (64\%). He was pale, weak, sweating, and febrile $\left(38.5^{\circ} \mathrm{C}\right)$. His pulse rate was $104 / \mathrm{min}$, blood pressure $110 / 50$ $\mathrm{mm} \mathrm{Hg}$, and respiratory rate $26 / \mathrm{min}$. A chest radiograph showed diffuse fluffy infiltrates predominantly in the lower lobes. The erythrocyte sedimentation rate was $80 \mathrm{~mm}$ in one 